USM-TH-113

\title{
Azimuthal Spin Asymmetries of Pion Electroproduction
}

\author{
Bo-Qiang Ma ${ }^{a}$, Ivan Schmidt ${ }^{b}$, and Jian-Jun Yang ${ }^{b, c}$ \\ ${ }^{a}$ Department of Physics, Peking University, Beijing 100871, China, \\ and CCAST (World Laboratory), P.O. Box 8730, Beijing 100080, China \\ ${ }^{b}$ Departamento de Física, Universidad Técnica Federico Santa María, \\ Casilla 110-V, Valparaíso, Chile \\ ${ }^{c}$ Department of Physics, Nanjing Normal University, Nanjing 210097, China
}

\begin{abstract}
Azimuthal spin asymmetries, both for charged and neutral pion production in semiinclusive deep inelastic scattering of unpolarized charged lepton beams on longitudinally and transversely polarized nucleon targets, are analyzed and calculated. Various assumptions and approximations in the quark distributions and fragmentation functions often used in these calculations are studied in detail. It is found that different approaches to the distribution and fragmentation functions may lead to quite different predictions on the azimuthal asymmetries measured in the HERMES experiments, thus their effects should be taken into account before using the available data as a measurement of quark transversity distributions. It is also found that the unfavored quark to pion fragmentation functions must be taken into account for $\pi^{-}$production from a proton target, although they can be neglected for $\pi^{+}$and $\pi^{0}$ production. Pion production from a proton target is suitable to study the $u$ quark transversity distribution, whereas a combination of pion production from both proton and neutron targets can measure the flavor structure of quark transversity distributions.
\end{abstract}

PACS numbers: 13.87.Fh, 13.60.-r, 13.88.+e, 14.20.Dh 


\section{Introduction}

Recently, the HERMES collaboration reported the observation of single-spin azimuthal asymmetries for charged and neutral pion production, in semi-inclusive deep inelastic scattering (DIS) of unpolarized positron beam on the longitudinally polarized proton target [1, 2]. Such azimuthal asymmetries are important, because they can provide information of the transversity distributions [3], which are one of the three fundamental quark distributions of the nucleon. The other two fundamental quark distributions are the momentum and helicity distributions, which have been explored for more than three decades in various DIS processes [4, 5], and their explicit flavordependence has been obtained with relative high precision, although there are still some uncertainties concerning the sea content at small Bjorken variable $x$ and the valence flavor-spin structure as $x \rightarrow 1$. However, the experimental measurements of the quark transversity distributions are just about to begin, since the transversity is not directly observable in inclusive DIS processes. It has been proposed that the transversity can manifest itself through the Collins effect [6] of nonzero production between a chiral-odd structure function and a T-odd fragmentation function, which is accessible in some specific semi-inclusive hadron production experiments [6, 7, 8, 9, 10, 11, 12]. Indeed, there have been a number of studies [13, 14, 15, 16, 17, 18, 19, 20, 21, 22, 23, 24] to show that the azimuthal asymmetries measured by HERMES can be used to extract the quark transversity distributions of the nucleon, although the theoretical predictions of the explicit flavor-dependent transversity distributions are different in various models [3]. Therefore the HERMES measurements provide us the opportunity to confront theoretical predictions with physical observations, and it is meaningful to examine the theoretical results carefully, in order to see what conclusion we can draw on the transversity.

The analyzing power of azimuthal spin asymmetry measured by HERMES is defined as

$$
A_{U L}^{W}=\frac{\int[\mathrm{d} \phi] W(\phi)\left\{N^{+}(\phi)-N^{-}(\phi)\right\}}{\frac{1}{2} \int[\mathrm{d} \phi]\left\{N^{+}(\phi)+N^{-}(\phi)\right\}},
$$

where $U L$ denotes unpolarized beam on a longitudinally polarized target, $W(\phi)=\sin \phi$ or $\sin 2 \phi$ is the weighting function for picking up the Collins effect, and $N^{+}(\phi)\left(N^{-}(\phi)\right)$ 
is the number of events for pion production, as a function of $\phi$, when the target is positively (negatively) polarized. The azimuthal angle $\phi$ is the angle between the pion emitting plane and the lepton scattering plane, with the lepton scattering plane determined by the incident and scattered leptons, and the pion emitting plane determined by the final detected pion and the virtual photon. The virtual photon acts as the common axis of both planes. The analyzing powers of azimuthal asymmetries for charged pions, $\pi^{+}$and $\pi^{-}$, and neutral pion, $\pi^{0}$, have been measured [1], 2], and there is clear evidence for non-zero values of $A_{U L}^{\sin \phi}$ for $\pi^{+}$and $\pi^{0}$ production, which indicates the existence of azimuthal asymmetries. Under a number of simplifying assumptions and approximations, the analyzing power $A_{U L}^{\sin \phi}$ of azimuthal asymmetry is proportional to the ratio

$$
\frac{\sum_{q} e_{q}^{2} \delta q(x) H_{1}^{\perp(1) q}(z)}{\sum_{q} e_{q}^{2} q(x) D_{1}^{q}(z)}
$$

Here $e_{q}$ is the charge of the quark with flavor $q, q(x)$ and $\delta q(x)$ are the quark momentum and transversity distributions of the nucleon target, $D_{1}^{q}(z)$ is the fragmentation function for an unpolarized quark with flavor $a$ into a pion $\pi$, and $H_{1}^{\perp(1) q}(z)$ is the so called Collins function describing the fragmentation of a transversely polarized quark into a pion $\pi$. If one assumes, as adopted in almost all previous analysis, that the pion production is dominated by the "favored" fragmentation processes $u \rightarrow \pi^{+}$ and $d \rightarrow \pi^{-}$for charged pions, and neglects the "unfavored" processes $u \rightarrow \pi^{-}$and $d \rightarrow \pi^{+}$, one may conclude that the $\pi^{+}$azimuthal asymmetry measures the $u$-quark transversity $\delta u(x)$ and the $\pi^{-}$azimuthal asymmetry measures the $d$-quark transversity $\delta d(x)$, respectively. This is the situation of the current available studies.

The purpose of this paper is to take into account the terms neglected in (2), and also to take into consideration the contribution from the "unfavored" fragmentation process. We will estimate their effects on the azimuthal asymmetries for the three kinds of produced pions: $\pi^{+}, \pi^{-}$, and $\pi^{0}$. We find that the neglected terms may have sizable effects on the azimuthal asymmetries in the HERMES kinematical region, and that the results are sensitive to different assumptions on the quark distributions and fragmentation functions. We find also that, although the neglect of the "unfavored" fragmentation processes seems to be reasonable for $\pi^{+}$production, it is not justi- 
fied for $\pi^{-}$production. Moreover, a significant difference between the $\pi^{-}$azimuthal asymmetries predicted by two different models [20], are largely reduced by taking into account the "unfavored" processes, and this renders it difficult to use $\pi^{-}$production as a clean process to test different predictions on the flavor-spin quark structure of the nucleon at large $x$. We will also check the possible ambiguities from the uncertainties in the "unfavored" fragmentation functions. However, it will be shown that

the favored fragmentation process in $\pi^{+}$production from a neutron target plays a more important role than that in the corresponding $\pi^{-}$production from a proton target. Thus $\pi^{+}$production from a neutron target can provide a test of different model predictions. A combination of pion production from both proton and neutron targets can measure both of the $u$ and $d$ quark transversity distributions, $\delta u(x)$ and $\delta d(x)$, of the proton.

This paper is organized as follows. In Section II of the paper, we will present the formulae for the azimuthal asymmetries, and analyze in details various assumptions and approximations on the quark distributions and fragmentation functions used for numerical calculations. In section III, we perform numerical calculations of the azimuthal asymmetries for pions: $\pi^{+}, \pi^{-}$, and $\pi^{0}$, and give detailed discussions on the effects of using different approaches to the distribution functions and fragmentation functions. We discuss also in detail the uncertainties coming from the "unfavored" processes of quark to pion fragmentation. In Section IV, we predict the azimuthal asymmetries for pion production in semi-inclusive DIS processes of an unpolarized charged lepton beam on a transversely polarized nucleon target. Finally, we present the summary and conclusions in Section V.

\section{Formalism of Azimuthal Spin Asymmetry in Pion Electroproduction}

Deep inelastic scattering (DIS) of a lepton on a nucleon is one of the most efficient and clean tools to detect the underlying structure of the nucleon. With the recent progress in experimental techniques, the precision detection of hadrons produced in 
DIS processes makes it possible to reveal more about the basic structure of the nucleon, and the use of polarized beams and/or polarized targets makes it also possible to measure with some precision various polarized quark distributions and fragmentation functions. The HERMES experiments [1, 2] are performed with an unpolarized lepton beam scattering on a longitudinally polarized proton target, and some assumptions and approximations are needed in order to connect the pion production with the transversity distribution, which is supposed to be related to the probability of finding a quark with its spin aligned to the proton spin for a transversity polarized proton. We first give the formulae for the analyzing power of azimuthal asymmetry and analyze the quantities needed to perform numerical calculations.

\subsection{Spin asymmetries of pion electroproduction}

We now introduce the basic kinematics for semi-inclusive DIS processes. Let $l_{1}$ and $l_{2}$ be the initial and final momenta of the incoming and outgoing charged leptons respectively, $Q^{2}=-q^{2}$ be the squared 4-momentum transfer of the virtual photon with momentum $q=l_{1}-l_{2}$, and $P$ and $P_{h}\left(M\right.$ and $\left.M_{h}\right)$ be the target and final hadron momenta (masses). Then the three basic variables, $x, y$, and $z$, used in describing DIS processes can be expressed as

$$
x=\frac{Q^{2}}{2 P \cdot q} ; \quad y=\frac{P \cdot q}{P \cdot l_{1}} ; \quad z=\frac{P \cdot P_{h}}{P \cdot q},
$$

where $x$ is the Bjorken variable which corresponds to the momentum fraction carried by the struck quark in a light-cone description, $y$ is the fraction of the initial lepton's energy transferred to the quark by the virtual photon, and $z$ is the fraction of quark momentum transferred to the final produced hadron in the fragmentation process. In the target rest frame, the three variables can be expressed as

$$
x=\frac{Q^{2}}{2 M \nu} ; \quad y=\frac{\nu}{E} ; \quad z=\frac{E_{h}}{\nu},
$$

where $\nu, E$, and $E_{h}$ are the virtual photon energy, the incident lepton energy, and the produced hadron energy, respectively. $s=2 P \cdot l=2 M E$ is the total squared energy in the lepton-proton center of mass frame, therefore we have a relation $Q^{2}=s x y$. The 
incident lepton energy is $E=27.6 \mathrm{GeV}$ in the HERMES experiments. The invariant mass of the photon-proton system is $W=\sqrt{2 M \nu+M^{2}-Q^{2}}$. Let $P_{h \perp}$ be the final hadron transverse momentum and $l_{1 \perp}$ be the initial lepton transverse momentum, both respect to the virtual photon momentum direction. Then the azimuthal angle $\phi$ is the angle between $P_{h \perp}$ and $l_{1 \perp}$ around the virtual photon direction.

Using the above measurable variables of semi-inclusive hadron production and the measurable cross sections, one can defined $\sin \phi$ and $\sin 2 \phi$ weighted spin asymmetries

$$
\begin{gathered}
\left\langle\frac{\left|P_{h \perp}\right|}{M_{h}} \sin \phi\right\rangle=\frac{\int \mathrm{d}^{2} P_{h \perp} \frac{\left|P_{h \perp}\right|}{M_{h}} \sin \phi\left(\mathrm{d} \sigma^{+}-\mathrm{d} \sigma^{-}\right)}{\int d^{2} P_{h \perp}\left(\mathrm{d} \sigma^{+}+\mathrm{d} \sigma^{-}\right)}, \\
\left\langle\frac{\left|P_{h \perp}\right|^{2}}{M M_{h}} \sin 2 \phi\right\rangle=\frac{\int \mathrm{d}^{2} P_{h T} \frac{\left|P_{h T}\right|^{2}}{M M_{h}} \sin 2 \phi\left(\mathrm{d} \sigma^{+}-\mathrm{d} \sigma^{-}\right)}{\int \mathrm{d}^{2} P_{h \perp}\left(\mathrm{d} \sigma^{+}+\mathrm{d} \sigma^{-}\right)},
\end{gathered}
$$

which are measurable quantities. Here $+(-)$ denotes parallel (antiparallel) longitudinal polarization of the target. The above asymmetries are related to the azimuthal asymmetries measured by HERMES through the following relations

$$
\begin{gathered}
A_{U L}^{\sin \phi} \approx \frac{2 M_{h}}{\left\langle P_{h \perp}\right\rangle}\left\langle\frac{\left|P_{h \perp}\right|}{M_{h}} \sin \phi\right\rangle, \\
A_{U L}^{\sin 2 \phi} \approx \frac{2 M M_{h}}{\left\langle P_{h \perp}^{2}\right\rangle}\left\langle\frac{\left|P_{h \perp}\right|^{2}}{M M_{h}} \sin 2 \phi\right\rangle .
\end{gathered}
$$

In the HERMES experiments, $\left\langle P_{h \perp}\right\rangle=0.44 \mathrm{GeV}$, and we may use $\left\langle P_{h \perp}^{2}\right\rangle=(0.44)^{2}$ $\mathrm{GeV}^{2}$ as an estimate of $\left\langle P_{h \perp}^{2}\right\rangle$ in our calculations.

From another side, one can use the quark-parton model to calculate hadron production in semi-inclusive DIS processes, and express the cross sections in terms of various distribution and fragmentation functions. Then one can define the weighted cross section for one flavor quark, $d \sigma_{q}$, with different weighting functions depending on the final hadron transverse momenta $w_{i}\left(P_{h \perp}\right)$ :

$$
\Sigma_{i}^{q}=\int \mathrm{d}^{2} P_{h \perp} w_{i}\left(P_{h \perp}\right) \mathrm{d} \sigma_{q}
$$

where $w_{i}\left(P_{h \perp}\right)=1, w_{i}\left(P_{h \perp}\right)=P_{h \perp} \sin \phi / M_{h}$, and $w_{i}\left(P_{h \perp}\right)=\left|P_{h \perp}\right|^{2} \sin 2 \phi / M M_{h}$. Thus one can relate the theoretical calculations with the above spin asymmetries by

$$
\left\langle\frac{\left|P_{h \perp}\right|}{M_{h}} \sin \phi\right\rangle=\frac{\Sigma_{2}}{\Sigma_{1}},
$$




$$
\left\langle\frac{\left|P_{h \perp}\right|^{2}}{M M_{h}} \sin 2 \phi\right\rangle=\frac{\Sigma_{3}}{\Sigma_{1}}
$$

where a sum over all quark flavors, $\Sigma_{i}=\sum_{q} e_{q}^{2} \Sigma_{i}^{q}$, is implicitly assumed, and will be assumed from now on. In the case of unpolarized beam and longitudinal polarized target, $\Sigma_{1}, \Sigma_{2}=\Sigma_{2 L}+\Sigma_{2 T}$, and $\Sigma_{3}$ are given by [4, 9, 21, 22]

$$
\begin{gathered}
\Sigma_{1}=\left[1+(1-y)^{2}\right] f_{1}(x) D_{1}(z), \\
\Sigma_{2 L}=4 S_{L} \frac{M}{Q}(2-y) \sqrt{1-y}\left[x h_{L}(x) z H_{1}^{\perp(1)}(z)-h_{1 L}^{\perp(1)}(x) \tilde{H}(z)\right], \\
\Sigma_{2 T}=2 S_{T x}(1-y) h_{1}(x) z H_{1}^{\perp(1)}(z), \\
\Sigma_{3}=8 S_{L}(1-y) h_{1 L}^{\perp(1)}(x) z^{2} H_{1}^{\perp(1)}(z) .
\end{gathered}
$$

Here the components of the longitudinal and transverse target polarization in the virtual photon frame are denoted by $S_{L}$ and $S_{T x}$, respectively:

$$
S_{L}=S \cos \theta_{\gamma}, \quad S_{T x}=S \sin \theta_{\gamma}
$$

with

$$
\sin \theta_{\gamma}=\sqrt{\frac{4 M^{2} x^{2}}{Q^{2}+4 M^{2} x^{2}}\left(1-y-\frac{M^{2} x^{2} y^{2}}{Q^{2}}\right)}=\sqrt{\frac{4 M^{2} x}{s y+4 M^{2} x}\left(1-y-\frac{M^{2} x y}{s}\right)},
$$

and $S$ is the target polarization, which has the value 0.86 in the HERMES experiments. Twist-2 distribution functions and fragmentation functions have a subscribe "1": $f_{1}$ and $D_{1}$ are the usual unpolarized distribution and fragmentation function, while $h_{1 L}^{\perp(1)}(x)$ and $h_{1}(x)$ are the quark transverse spin distribution functions of longitudinally and transversely polarized nucleons, respectively. $h_{L}(x)$ is the twist-3 distribution function of a longitudinally polarized nucleon, and it can be split into a twist-2 part, $h_{1 L}^{\perp(1)}(x)$, and an interaction dependent part, $\tilde{h}_{L}(x)$ :

$$
h_{L}(x)=-2 \frac{h_{1 L}^{\perp(1)}(x)}{x}+\tilde{h}_{L}(x) .
$$

The fragmentation function $\tilde{H}(z)$ is the interaction dependent part of the twist-3 fragmentation function:

$$
H(z)=-2 z H_{1}^{\perp(1)}(z)+\tilde{H}(z) .
$$


The functions with superscript “(1)" denote $p_{\perp}^{2}$ - and $k_{\perp}^{2}$-moments, respectively:

$$
\begin{gathered}
h_{1 L}^{\perp(1)}(x) \equiv \int \mathrm{d}^{2} p_{\perp} \frac{p_{\perp}^{2}}{2 M^{2}} h_{1 L}^{\perp}\left(x, p_{\perp}^{2}\right), \\
H_{1 L}^{\perp(1)}(z) \equiv z^{2} \int \mathrm{d}^{2} k_{\perp} \frac{k_{\perp}^{2}}{2 M_{h}^{2}} H_{1 L}^{\perp}\left(z, z^{2} k_{\perp}^{2}\right),
\end{gathered}
$$

where $p_{\perp}$ and $k_{\perp}$ are the intrinsic transverse momenta of the initial and final partons in the target and produced hadrons, respectively.

To calculate the spin asymmetries and compare them with experiments, we need to know the quark distribution functions: $f_{1}(x), h_{1}(x), \tilde{h}_{L}(x)$, and $h_{1 L}^{\perp(1)}(x)$, and the fragmentation functions: $D_{1}(z), H_{1}^{\perp(1)}(z)$, and $\tilde{H}(z)$. Unfortunately, most of the distribution functions and fragmentation functions which appear in these expressions are not known a priori, since they have not been measured yet. Thus we have to make some assumptions and approximations, and this leads to different approaches for the distribution functions and fragmentation functions [17, 22].

\subsection{Different approaches to the distribution functions and fragmentation functions}

The most simple approach, denoted as Leading Approach, is to neglect the $1 / Q$ term $\Sigma_{2 L}$ in $\Sigma_{2}$, i.e., we neglect both the $\tilde{h}_{L}(x)$ and $h_{1 L}^{\perp(1)}(x)$ terms in the spin asymmetry $\left\langle\frac{\left|P_{h \perp}\right|}{M_{h}} \sin \phi\right\rangle$. Then we find immediately that

$$
\left\langle\frac{\left|P_{h \perp}\right|}{M_{h}} \sin \phi\right\rangle=\frac{\Sigma_{2 T}}{\Sigma_{1}} \propto \frac{h_{1}(x) H_{1}^{\perp(1)}(z)}{f_{1}(x) D_{1}(z)},
$$

which is Eq. (2). Thus we can relate the quark transversity distribution $h_{1}(x)$ to the spin asymmetry with its $x$-dependence. This is the situation discussed in several papers [14, 20].

The next approach, denoted as Approach 1, is to assume that the twist-2 quark transverse spin distribution function of longitudinally polarized nucleon, $h_{1 L}^{\perp(1)}(x)$, is zero [22]. Then it follows that

$$
h_{L}(x)=\tilde{h}_{L}(x)=h_{1}(x)
$$


Another approach, denoted as Approach 2, is to assume that the interaction dependent twist-3 part, $\tilde{h}_{L}(x)$, is zero [13], and consequently, we can also assume that $\tilde{H}(z)$ is zero. Then by neglecting the term proportional to the current quark mass, one can obtain a Wandzura-Wilczek type relation 25, 26]

$$
h_{1 L}^{\perp(1)}(x)=-x^{2} \int_{x}^{1} \mathrm{~d} \xi \frac{h_{1}(\xi)}{\xi^{2}} .
$$

It follows, from Eq. (18), that

$$
h_{L}(x)=2 x \int_{x}^{1} \mathrm{~d} \xi \frac{h_{1}(\xi)}{\xi^{2}} .
$$

In the above two approaches, we only need the distribution functions, $f_{1}(x)$ and $h_{1}(x)$, and the fragmentation functions, $D_{1}(z)$ and $H_{1}^{\perp(1)}(z)$, in order to calculate the spin asymmetries $\left\langle\frac{\left|P_{h \perp}\right|}{M_{h}} \sin \phi\right\rangle$ and $\left\langle\frac{\left|P_{h}\right|^{2}}{M M_{h}} \sin 2 \phi\right\rangle$.

\subsection{Quark transversity distributions of the nucleon}

In the three approaches described above, we need the quark distribution functions, $f_{1}(x)=q(x)$ and $h_{1}(x)=\delta q(x)$, as inputs. The $x$-dependence of the spin asymmetries are controlled by the above two quark distribution functions, in which the unpolarized quark distributions $q(x)$ are known to relative high precision, thus we are able to extract the information on the quark transversity distributions $\delta q(x)$ by comparing theoretical predictions with the experimental data on spin asymmetries.

Although the quark momentum distributions $q(x)$ have been measured to high precision, there are still uncertainties concerning the flavor structure as $x \rightarrow 1$. For example, the SU(6) quark spectator diquark model [27, 28, 29] predicts $d(x) /\left.u(x)\right|_{x=1}=0$, whereas a pQCD based counting rule analysis [30, 31, 32] predicts $d(x) /\left.u(x)\right|_{x=1}=$ $1 / 5$. The flavor structure of the transversity distributions in the two models are also found to differ significantly in this region 20, 33]: the pQCD based analysis predicts [20, 33] $\delta d(x) / d(x) \rightarrow 1$, whereas the $\mathrm{SU}(6)$ quark-spectator-diquark model predicts [34, 35] $\delta d(x) / d(x) \rightarrow-1 / 3$. Detailed constructions of the quark transversity distributions in the two models can be found in Refs. 20, 33.

To make realistic predictions of measurable quantities, we need also to take into account the sea quark contribution in the two model constructions. In the quark 
diquark model case, this can be achieved by adopting one set of unpolarized quark distribution parametrization as input, and then use theoretical relations to connect the quark transversity distributions with the unpolarized quark distributions [29, 34]:

$$
\begin{gathered}
\delta u_{v}(x)=\left[u_{v}(x)-\frac{1}{2} d_{v}(x)\right] \hat{W}_{S}(x)-\frac{1}{6} d_{v}(x) \hat{W}_{V}(x) \\
\delta d_{v}(x)=-\frac{1}{3} d_{v}(x) \hat{W}_{V}(x),
\end{gathered}
$$

in a similar way as was done for the quark helicity distributions [29]. $\hat{W}_{S}(x)$ and $\hat{W}_{S}(x)$, which come from the relativistic effect of quark transversal motions [36], are the Melosh-Wigner rotation factors [34, 35, 37] for spectator scalar and vector diquarks. We use the valence quark momentum distributions $u_{v}(x)$ and $d_{v}(x)$ from quark distribution parametrization, but with $\hat{W}_{S}(x)$ and $\hat{W}_{V}(x)$ from the model calculation [34]. In this way we can take into account the sea contribution for the unpolarized quark distributions through the input parametrization. This can provide a more reliable prediction for the absolute magnitude and shape of a physical quantity than directly from the model calculation.

For the pQCD based analysis, we adopt the same consideration as above and make the following connection to relate the pQCD model quark distributions with the parametrization

$$
\begin{gathered}
u_{v}^{p Q C D}(x)=u_{v}^{\text {para }}(x), \quad d_{v}^{p Q C D}(x)=\frac{d_{v}^{\text {th }}(x)}{u_{v}^{\text {th }}(x)} u_{v}^{\text {para }}(x), \\
\delta u_{v}^{p Q C D}(x)=\frac{\delta u_{v}^{t h}(x)}{u_{v}^{t h}(x)} u_{v}^{\text {para }}(x), \quad \delta d_{v}^{p Q C D}(x)=\frac{\delta d_{v}^{\text {th }}(x)}{u_{v}^{\text {th }}(x)} u_{v}^{\text {para }}(x),
\end{gathered}
$$

where the superscripts "th" means the pure theoretical calculation in the pQCD analysis [20, 33], and "para" means the input from parametrization. The superscript "pQCD" means that the new quark distributions keep exactly the same flavor and spin structure as that in the pQCD analysis, but with detailed $x$-dependent behaviors more closed to the realistic situation. This is equivalent to use an unique correction factor, $u_{v}^{\text {para }}(x) / u_{v}^{t h}$, to adjust each pure theoretically calculated quantity to a more realistic pQCD model quantity. In this way we can take into account the sea contribution by using the sea quark distributions from parametrization, while still keep the pQCD model behaviors of the valence quark distributions. 

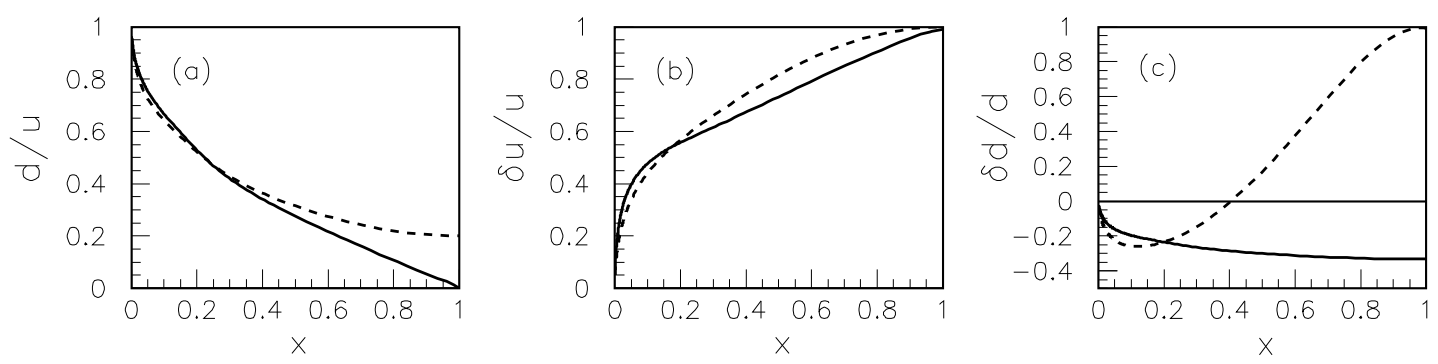

Figure 1: The different flavor and transversity structure of the two model quark distributions: (a) $d_{v}(x) / u_{v}(x)$; (b) $\delta u_{v}(x) / u_{v}(x)$; and (c) $\delta d_{v}(x) / d_{v}(x)$. The solid and dashed curves correspond to the quark-diquark model and the pQCD analysis, respectively.

The explicit flavor and transversity structure of the two sets of quark distributions can be found in Fig. 1. Thus we have two sets of quark distributions of $q(x)$ and $\delta q(x)$, which keeps the same valence behaviors as in the quark diquark model and pQCD based model predictions, but with the sea from parametrization. We expect to make some more realistic predictions based on these two sets of quark distributions, while still can distinguish between the different flavor and spin structure predicted by the two models.

There might be some uncertainties of the magnitude of the explicit quark distributions, however, these uncertainties will be reduced since we will only calculate quantities which involve the ratios of different quark distributions in our later numerical calculations.

\subsection{Fragmentation functions of pion}

We now turn our attention to the fragmentation functions $D_{1}(z)$ and $H_{1}^{\perp(1)}(z) . D_{1}(z)$ is the unpolarized fragmentation function for a quark splitting into a pion, and in previous studies on the azimuthal asymmetries, only the favored fragmentation functions, e.g., for a $u$ or $\bar{d}$ quark into $\pi^{+}$, are considered. However, a new parametrization of quark to pion fragmentation functions has been proposed [38], which combines pion production data from both semi-inclusive DIS and $e^{+} e^{-}$processes. This new parametrization provides a complete set of both favored and unfavored fragmenta- 
tion functions, and therefore it is very useful for the extraction of nucleon structure information from pion production in semi-inclusive DIS processes. In this paper, we will adopt this set of quark to pion fragmentation functions in our analysis of the transversity distributions through the azimuthal spin asymmetries discussed above.

For the favored fragmentation function, we mean the fragmentation function describing the transition of a "valence" quark into the pion, and it is, assuming isospin symmetry,

$$
D(z)=D_{u}^{\pi^{+}}(z)=D_{\frac{\pi^{+}}{d}}(z)=D_{d}^{\pi^{-}}(z)=D_{\bar{u}}^{\pi^{-}}(z)
$$

for charged pion fragmentation. The unfavored fragmentation function is supposed to describe the transition of a light-flavor "sea" quark into pions, and it is

$$
\hat{D}(z)=D_{\bar{u}}^{\pi^{+}}(z)=D_{d}^{\pi^{+}}(z)=D_{\frac{\pi^{-}}{d}}(z)=D_{u}^{\pi^{-}}(z)
$$

for $\pi^{ \pm}$fragmentation. For the case of the neutral pion, assuming that

$$
D_{q}^{\pi^{0}}(z)=\frac{1}{2}\left[D_{q}^{\pi^{+}}(z)+D_{q}^{\pi^{-}}(z)\right]
$$

we find

$$
D^{\pi^{0}}(z)=D_{u}^{\pi^{0}}(z)=D_{\bar{u}}^{\pi^{0}}(z)=D_{d}^{\pi^{0}}(z)=D_{\frac{\pi^{0}}{d}}(z)=\frac{1}{2}[D(z)+\hat{D}(z)] .
$$

The explicit analytically forms of $D(z)$ and $\hat{D}(z)$ are given by 38

$$
\begin{aligned}
& D(z)=0.689 z^{-1.039}(1-z)^{1.241}, \\
& \hat{D}(z)=0.217 z^{-1.805}(1-z)^{2.037} .
\end{aligned}
$$

From another point of view, assuming the Gribov-Lipatov relation connecting the fragmentation functions and the distribution functions [39, 40, 41], we have

$$
D_{q}^{\pi}(z) \propto z q^{\pi}(z)
$$

where $q_{\pi}(z)$ is the quark distribution of finding a quark $q$ with momentum fraction $x=z$ in the pion. We also know from the pQCD based analysis [31, 32] that the $x \rightarrow 1$ behavior of the quark distribution in the hadron $h$ satisfies the counting rule

$$
q^{h}(x) \propto(1-x)^{2 n_{\mathrm{s}}-1}
$$


where $n_{\mathrm{s}}$ means the spectator quark number. For favored quark fragmentation we notice that

$$
D(z) \propto(1-z)
$$

because there is only one spectator quark, whereas it is

$$
\hat{D}(z) \propto(1-z)^{5}
$$

for unfavored quark fragmentation because the spectator quark number $n_{\mathrm{s}}=3$ in this case. Thus we may assume

$$
\hat{D}(z)=(1-z)^{4} D(z)
$$

in this extreme situation. To reflect the possible uncertainties in the parametrization of fragmentation functions, we also assume three options for the relation between favored and unfavored fragmentation functions

$$
\hat{D}(z)=(1-z)^{n} D(z)
$$

with $n=2,3,4$, respectively, to compare with the new parametrization (33) described above.

The so called Collins fragmentation function $H_{1}^{\perp(1)}(z)$, which describes the transition of a transversely polarized quark into a pion, has not been systematically measured yet, and it is also theoretically not well known. However, there has been a so called Collins parametrization [6] of this fragmentation function, and we will adopt this parametrization in this paper. It is suggested that

$$
A_{C}\left(z, k_{\perp}\right)=\frac{\left|k_{\perp}\right| H_{1}^{\perp q}\left(z, z^{2} k_{\perp}^{2}\right)}{M_{h} D_{1}^{q}\left(z, z^{2} k_{\perp}^{2}\right)}=\frac{M_{C}\left|k_{\perp}\right|}{M_{C}^{2}+\left|k_{\perp}^{2}\right|},
$$

with $M_{C}$ being a typical hadronic scale around $0.3 \rightarrow 1 \mathrm{GeV}$. Assuming a Gaussian type of the quark transverse momentum dependence in the unpolarized fragmentation function

$$
D_{1}^{q}\left(z, z^{2} k_{\perp}^{2}\right)=D_{1}^{q}(z) \frac{R^{2}}{\pi z^{2}} \exp \left(-R^{2} k_{\perp}^{2}\right)
$$

one obtains

$$
H_{1}^{\perp(1) q}(z)=D_{1}^{q}(z) \frac{M_{C}}{2 M_{h}}\left(1-M_{C}^{2} R^{2} \int_{0}^{\infty} \mathrm{d} x \frac{\exp (-x)}{x+M_{C}^{2} R^{2}}\right),
$$


where $R^{2}=z^{2} /\left\langle P_{h \perp}^{2}\right\rangle$, and $\left\langle P_{h \perp}^{2}\right\rangle=z^{2}\left\langle k_{\perp}^{2}\right\rangle$ is the mean-square momentum the hadron acquires in the quark fragmentation process. We will set the parameters $M_{C}=0.7$ $\mathrm{GeV}$ and $\left\langle P_{h \perp}^{2}\right\rangle=(0.44)^{2} \mathrm{GeV}^{2}$ as they are consistent with the spin asymmetry measured at HERMES [23].

In fact, this fragmentation function controls the $z$ - and $P_{h \perp}$-dependence of the azimuthal asymmetries, therefore the $z$ - and $P_{h \perp}$-dependence will not be considered in this paper. We will mainly aim at the physics of the transversity distributions, which controls the $x$-dependence of the azimuthal asymmetries.

\section{Numerical Calculations}

With the kinematics of semi-inclusive process and the formulae for the azimuthal spin asymmetries of pion production, and also with the quark distribution functions and fragmentation functions given in the above section, we can perform numerical calculations under different assumptions and options. We will compare the numerical calculations of the azimuthal asymmetries with the HERMES experimental data [1], 2] constrained by the following experimental cuts

$$
1<Q^{2}<15 \mathrm{GeV}^{2}, \quad W>2 \mathrm{GeV}, \quad 0.2<y<0.85, \quad 0.2<z<0.7 \text {. }
$$

For the $Q^{2}$ and $W$ used in the integration over $y$ and $z$, we use the relations

$$
Q^{2}=s x y, \quad W^{2}=s y(1-x)+M^{2},
$$

with $s=2 M E=51.8 \mathrm{GeV}^{2}$ in the HERMES experiments. For the unpolarized quark distributions, we use the CTEQ parametrization [42] (CTEQ5 set 1) as input for both the quark diquark model and pQCD model quark distribution functions described in the above section.

\subsection{Different approaches of distribution functions and frag- mentation functions}

We first check the difference of the azimuthal asymmetries between different approaches of distribution functions and fragmentation functions. In this situation we 
take into account the contribution from both the favored and unfavored fragmentation functions, i.e., with the new parametrization of both $D(z)$ and $\hat{D}(z)$ in Eq. (33) as inputs. The numerical results for Leading Approach, Approach 1, and Approach 2 are given in Fig. 2. We find that both Approach 1 and Approach 2 differ significantly from Leading Approach, and this implies that contribution from the distribution functions $\tilde{h}_{L}(x)$ and $h_{1 L}^{\perp(1)}(x)$ are not negligible in the available HERMES experimental region. We also found that the magnitude of the azimuthal asymmetries from Leading Approach is below the data, thus Leading Approach cannot describe the available $\pi^{+}$ and $\pi^{0}$ data. However, both Approach 1 and Approach 2 with the two model transversity distributions are consistent with the available data, except for the data point at $x=0.26$ for $\pi^{-}$production. But the predictions from Approach 1 and Approach 2 have a large difference at large $x$, and we expect that further experimental data at large $x$ will be able to distinguish between the two approaches. We can also arrive at a following conclusion: although the HERMES azimuthal asymmetries for longitudinally polarized target [1, 2] can provide a rough estimate of the quark transversity distributions by comparing theoretical calculations with the experimental data, they do not make a direct measurement of the quark transversity distributions since the contribution from other quark distribution functions might be also sizable.

\subsection{Different cases of favored and unfavored fragmentation}

We next check the contribution from the unfavored fragmentation functions $\hat{D}(z)$. For this purpose we compare the calculations in two cases: with both favored and unfavored fragmentation functions as Case 1, and with only favored fragmentation functions as Case 2. In order to simplify the discussion, we will only perform calculations for Approach 2, and the results are given in Fig. 3. We find that there is almost no difference between the calculated results in the two cases for $\pi^{+}$and $\pi^{0}$ production, and this supports the favored fragmentation dominance assumption for the $\pi^{+}$ and $\pi^{0}$ production, as was adopted in the literature. However, the situation is quite different in the two cases for $\pi^{-}$production. This can be seen from Fig. 3 (c) and (f), where the calculated results for the two cases differ significantly. This implies that 

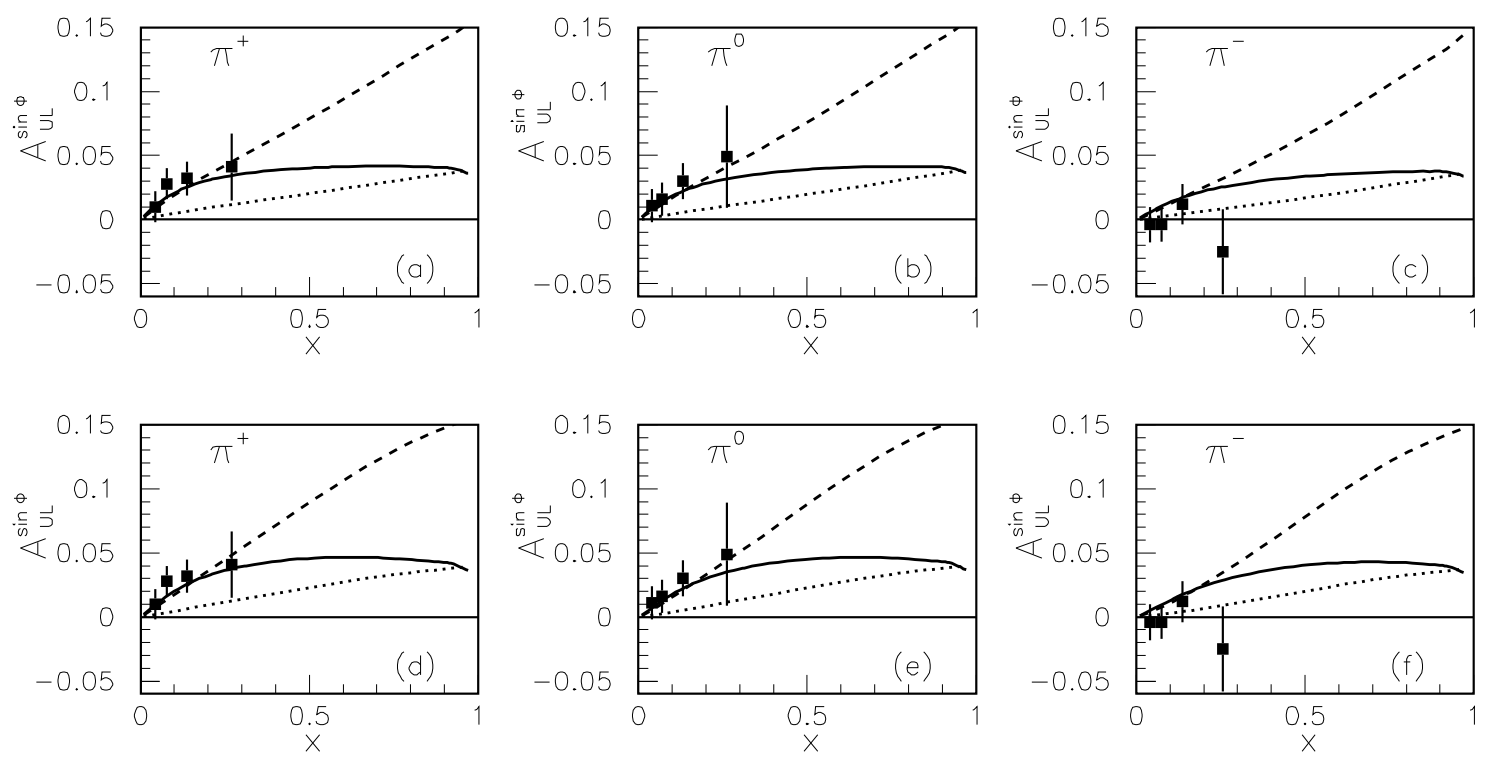

Figure 2: The azimuthal asymmetries $A_{U L}^{\sin \phi}$ for semi-inclusive $\pi^{+}, \pi^{0}$, and $\pi^{-}$production in deep inelastic scattering of unpolarized positron on the longitudinally polarized proton target. The upper row corresponds to (a) $\pi^{+}$, (b) $\pi^{0}$, and (c) $\pi^{-}$in the quark diquark model, and the lower row corresponds to (d) $\pi^{+}$, (e) $\pi^{0}$, and (f) $\pi^{-}$in the pQCD based analysis. The dotted, dashed, and solid curves correspond to the calculated results for Leading Approach, Approach 1, and Approach 2, respectively, of different assumptions of distribution functions and fragmentation functions. The data are taken from the HERMES experiments [1, 2].

the unfavored fragmentation functions have a large contribution to $\pi^{-}$production. This can be easily understood, since the most important unfavored process for $\pi^{-}$ production is $u \rightarrow \pi^{-}$. The big difference between Case 1 and Case 2 shows that the $u \rightarrow \pi^{-}$process is not negligible due to the $u$-dominance of the quark distributions in the proton target at large $x$.

It is interesting to notice that, although there is clear evidence for the non-zero azimuthal asymmetry for $\pi^{+}$and $\pi^{0}$ production, the available data for $\pi^{-}$production are consistent with no azimuthal asymmetry. The data are consistent with the calculated results with only favored fragmentation functions, i.e., Case 2 [14, 20]. However, the calculated results of Case 1 predict non-zero azimuthal asymmetry for $\pi^{-}$production in both of the quark diquark model and the pQCD based analysis. From Fig. 3 (c) and (f) we find that the data point for $\pi^{-}$production at $x=0.26$ 

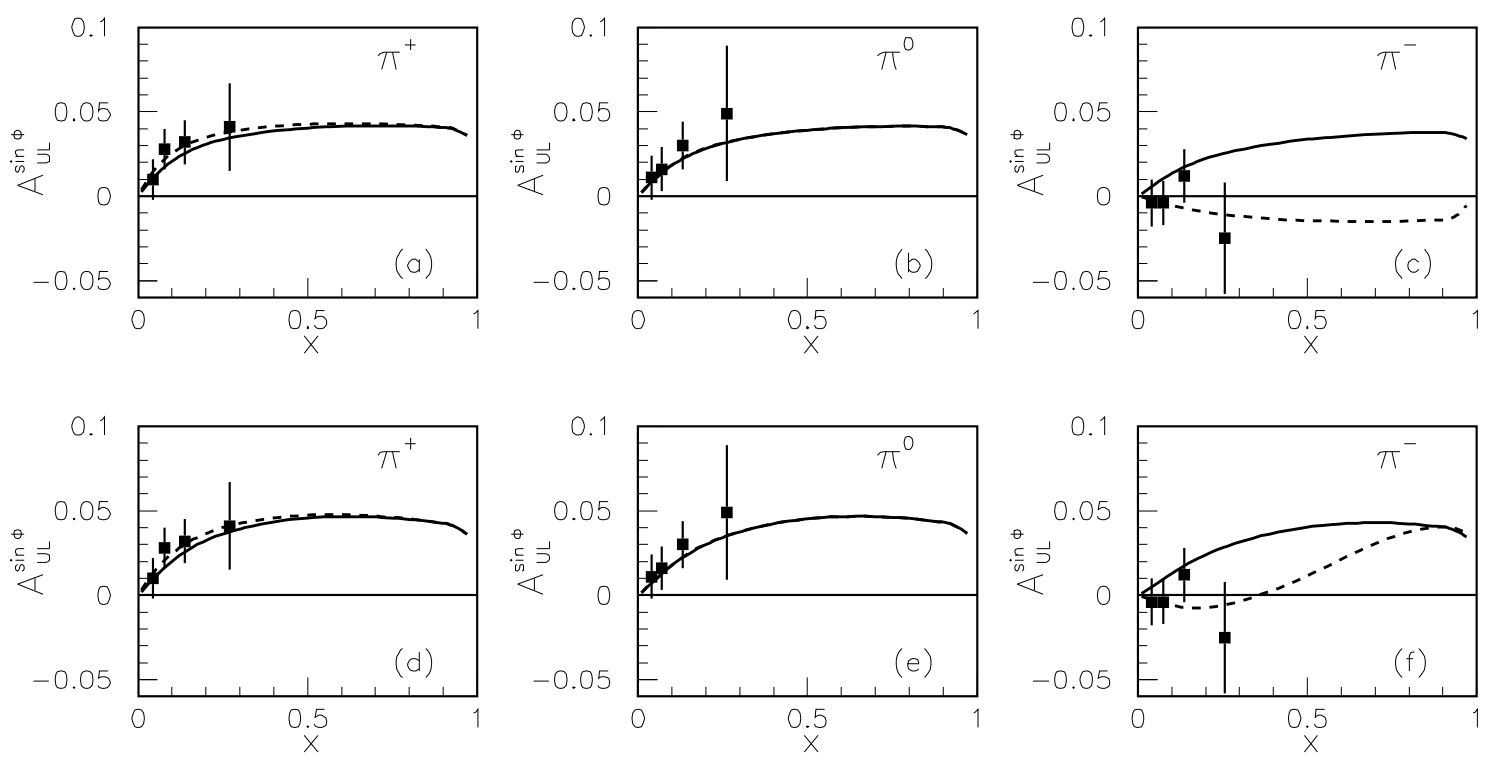

Figure 3: The azimuthal asymmetries $A_{U L}^{\sin \phi}$ for semi-inclusive $\pi^{+}, \pi^{0}$, and $\pi^{-}$production in deep inelastic scattering of unpolarized positron on the longitudinally polarized proton target. The upper row corresponds to (a) $\pi^{+}$, (b) $\pi^{0}$, and (c) $\pi^{-}$in the quark diquark model, and the lower row corresponds to (d) $\pi^{+}$, (e) $\pi^{0}$, and (f) $\pi^{-}$in the pQCD based analysis. The solid and dashed curves correspond to the calculated results for Case 1 with both favored and unfavored fragmentation and Case 2 with only favored fragmentation, respectively. The curves of the two cases overlap with each other for $\pi^{0}$ production.

seems to be in disagreement with this prediction. We suggest more analysis on $\pi^{-}$ production at the $x>0.2$ region in order to test this prediction.

We need to examine the possible ambiguities from the uncertainties of the unfavored fragmentation functions. For this purpose we choose the three options of $\hat{D}(z)=(1-z)^{n} D(z)$ with $n=2,3,4$, respectively, and present the calculated results in Fig. 团 with cuts $0.2<z<0.7$, and in Fig. 0 with cuts $0.6<z<0.8$, respectively. For the available data with cuts $0.2<z<0.7$, the three different options of the unfavored fragmentation functions do not have big difference, even for $\pi^{-}$production, as shown in Fig. 国. However, for the cuts with higher $z$, where the unfavored fragmentation is more suppressed than the favored ones for larger $n$, there is a large difference between the calculated results for $\pi^{-}$production with the three options, as shown in Fig. 5. Also there is a big difference between the predictions for $\pi^{-}$production with 
the two different models of transversity distributions. Therefore the $\pi^{-}$production at large $x$ and large $z$ is sensitive to the uncertainties of the unfavored fragmentation functions, and this is a region deserving careful experimental studies.
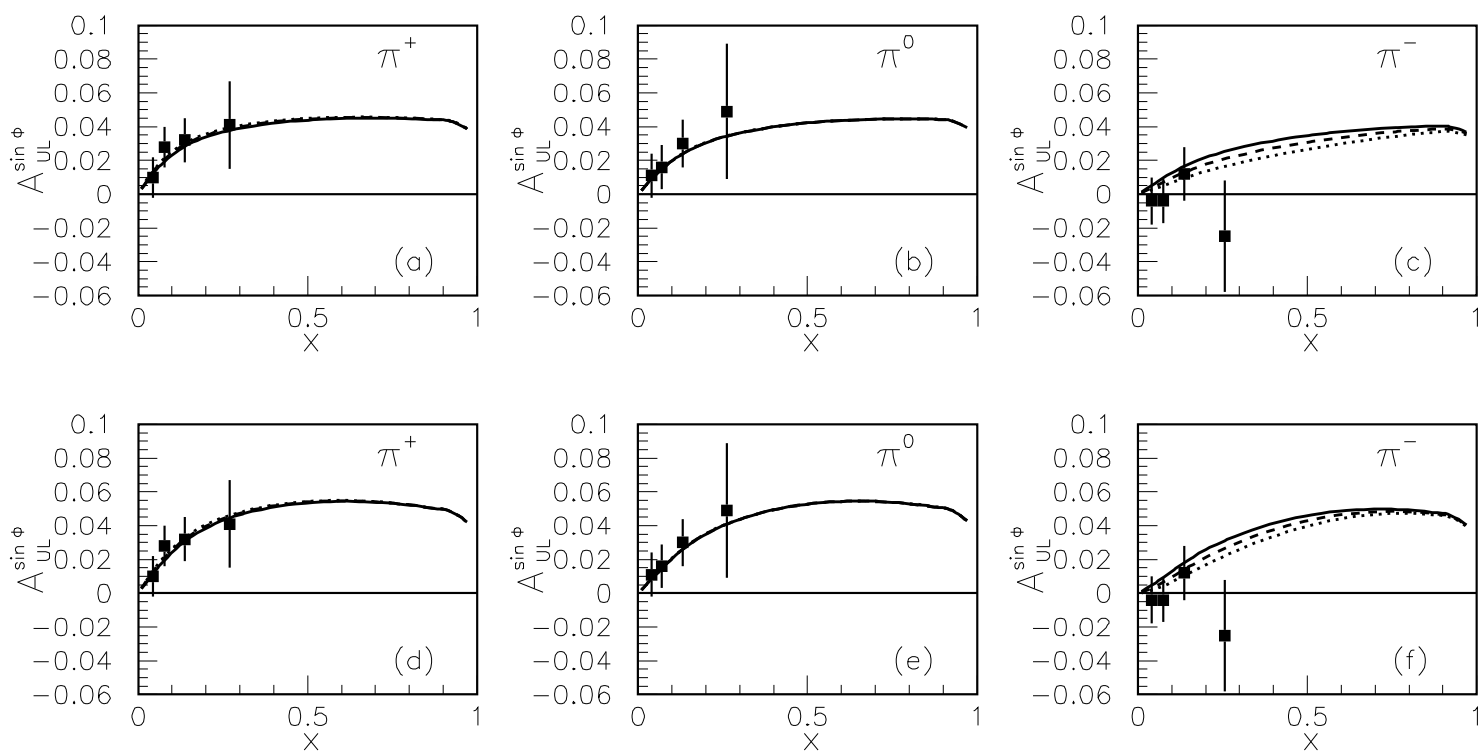

Figure 4: The azimuthal asymmetries $A_{U L}^{\sin \phi}$ for semi-inclusive $\pi^{+}, \pi^{0}$, and $\pi^{-}$production in deep inelastic scattering of unpolarized positron on the longitudinally polarized proton target. The upper row corresponds to (a) $\pi^{+}$, (b) $\pi^{0}$, and (c) $\pi^{-}$in the quark diquark model, and the lower row corresponds to (d) $\pi^{+}$, (e) $\pi^{0}$, and (f) $\pi^{-}$in the pQCD based analysis. The solid, dashed, and dotted curves correspond to the calculated results for three different options of unfavored fragmentation functions with $n=2,3,4$, respectively. The cuts for $z$ are $0.2<z<0.7$.

With the contribution from only the favored fragmentation functions considered, it is found 20 that there is a big difference between the azimuthal asymmetries for $\pi^{-}$production predicted by the quark diquark model and by the pQCD based analysis, as can be seen from the dashed curves in Fig. 3 (c) and (f). However, after taking into account the unfavored fragmentation processes, we find a much reduced difference between the two different model predictions, see the solid curves in Fig. 3 (c) and (f). Thus a clear distinction between the two different predictions will require very high precision measurement in the region with both large $x$ and large $z$ for the proton target, and this is difficulty to access experimentally. It also reminds us that 

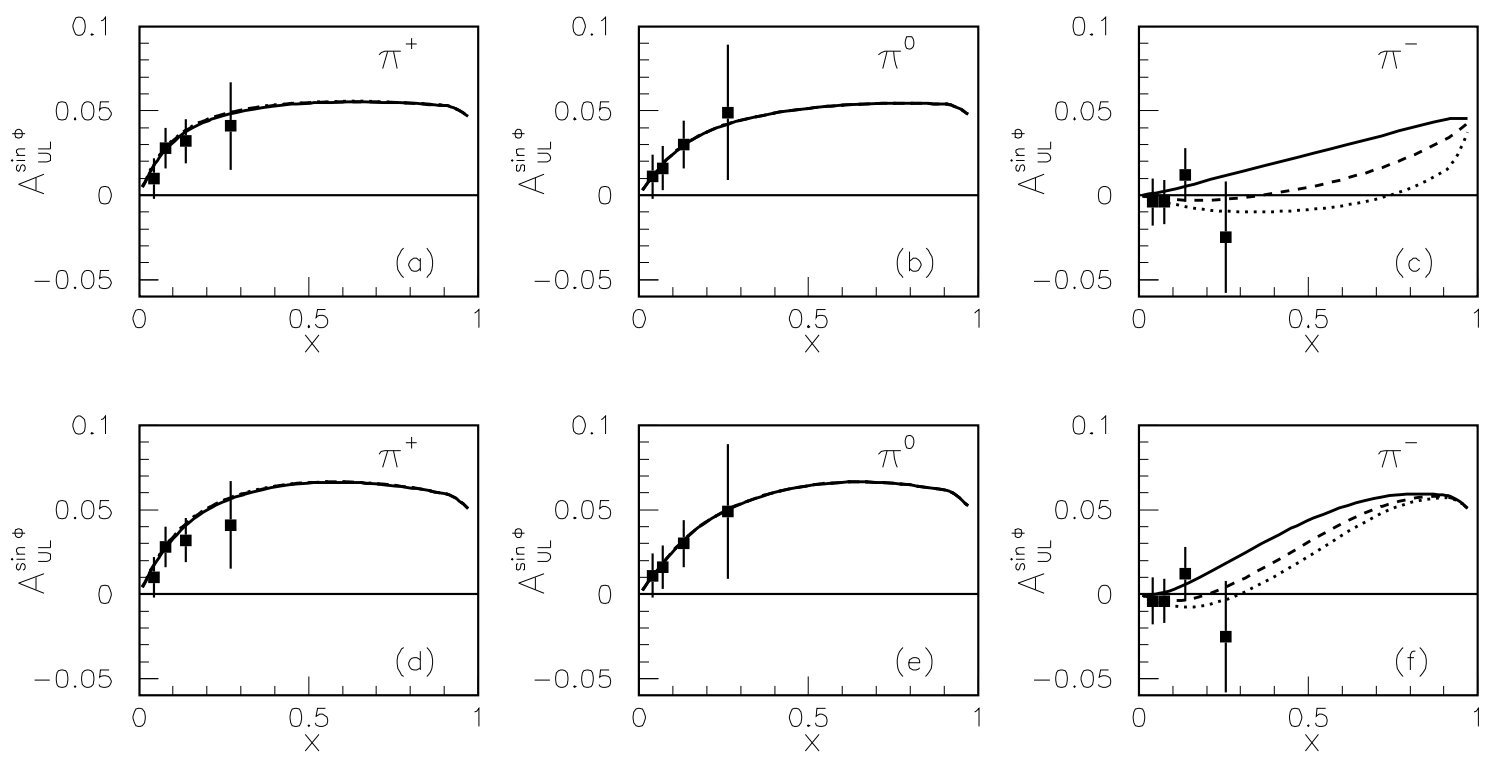

Figure 5: The same as Fig. 1 , but with cuts $0.6<z<0.8$.

the $\pi^{+}, \pi^{0}$, and $\pi^{-}$production from the proton target are mainly controlled by the $u$ quark fragmentation, due to the $u$ quark dominance in the proton target at large $x$. Thus the measured azimuthal asymmetries by the HERMES collaboration [1, 2] are mainly controlled by the $u$ quark transversity distribution $\delta u(x)$, and they are not sensitive to the $d$ quark transversity distribution $\delta d(x)$.

\subsection{Deuteron and neutron targets}

We now consider the possibility to distinguish between different models by using the deuteron as target, where there are equal numbers of $u$ and $d$ quarks from isopin symmetry. To calculate the azimuthal asymmetries for the deuteron target, we still use the formalism in Sec.II, with the target polarization $S_{D}=0.75$ multiplied by an correction factor

$$
\left(1-\frac{3}{2} \omega_{D}\right)
$$

where $\omega_{D}=0.05 \pm 0.01$ is the probability of the deuteron to be in the $D$-state. The azimutahl asymmetries for $\pi^{+}, \pi^{0}$, and $\pi^{-}$production are calculated for different approaches of distribution functions and fragmentation functions, and with the 
quark transversity distributions of the quark diquark model and the pQCD based analysis, respectively. For the unpolarized fragmentation functions, we use the new parametrization Eq. (33), and for the kinematical cuts we use the same as those in the HERMES experiments. The results are given in Fig. 6. From there we find that the deuteron is also not very sensitive to the two different sets of transversity distributions.
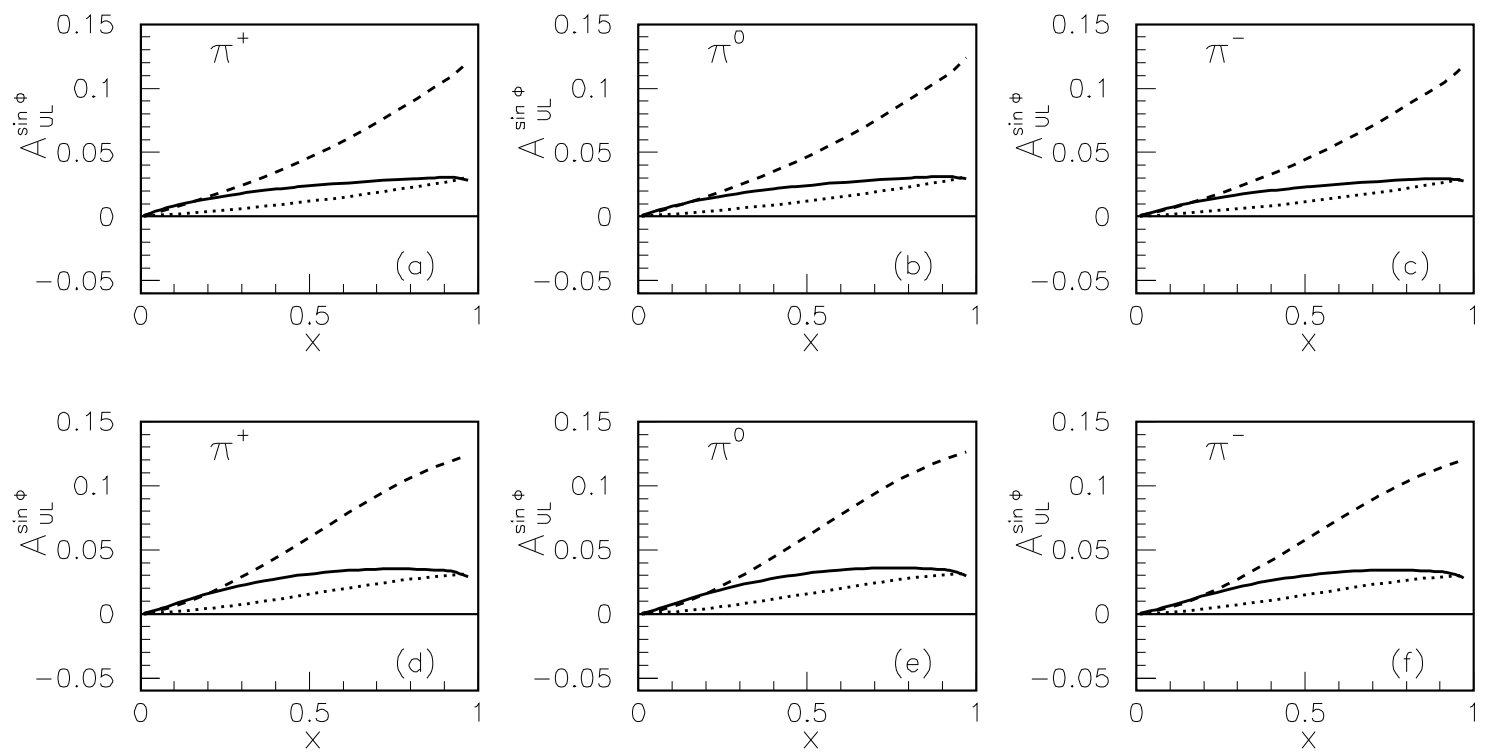

Figure 6: The azimuthal asymmetries $A_{U L}^{\sin \phi}$ for semi-inclusive $\pi^{+}, \pi^{0}$, and $\pi^{-}$production in deep inelastic scattering of unpolarized positron on the longitudinally polarized deuteron target. The upper row corresponds to (a) $\pi^{+}$, (b) $\pi^{0}$, and (c) $\pi^{-}$in the quark diquark model, and the lower row corresponds to (d) $\pi^{+}$, (e) $\pi^{0}$, and (f) $\pi^{-}$in the pQCD based analysis. The dotted, dashed, and solid curves correspond to the calculated results for Leading Approach, Approach 1, and Approach 2, respectively, of different assumptions of quark distributions and fragmentation functions.

We now check whether the situation can be improved if we use the neutron as target. This can be experimentally accessed by measuring the numerators and denominators of the spin asymmetries (10) from proton and deuteron targets respectively, and then extract the numerator and denominator for the neutron contribution to calculate the spin symmetry of the neutron. The calculated results are given in Fig. 7. We find that there is a somewhat bigger difference between the predictions of the 

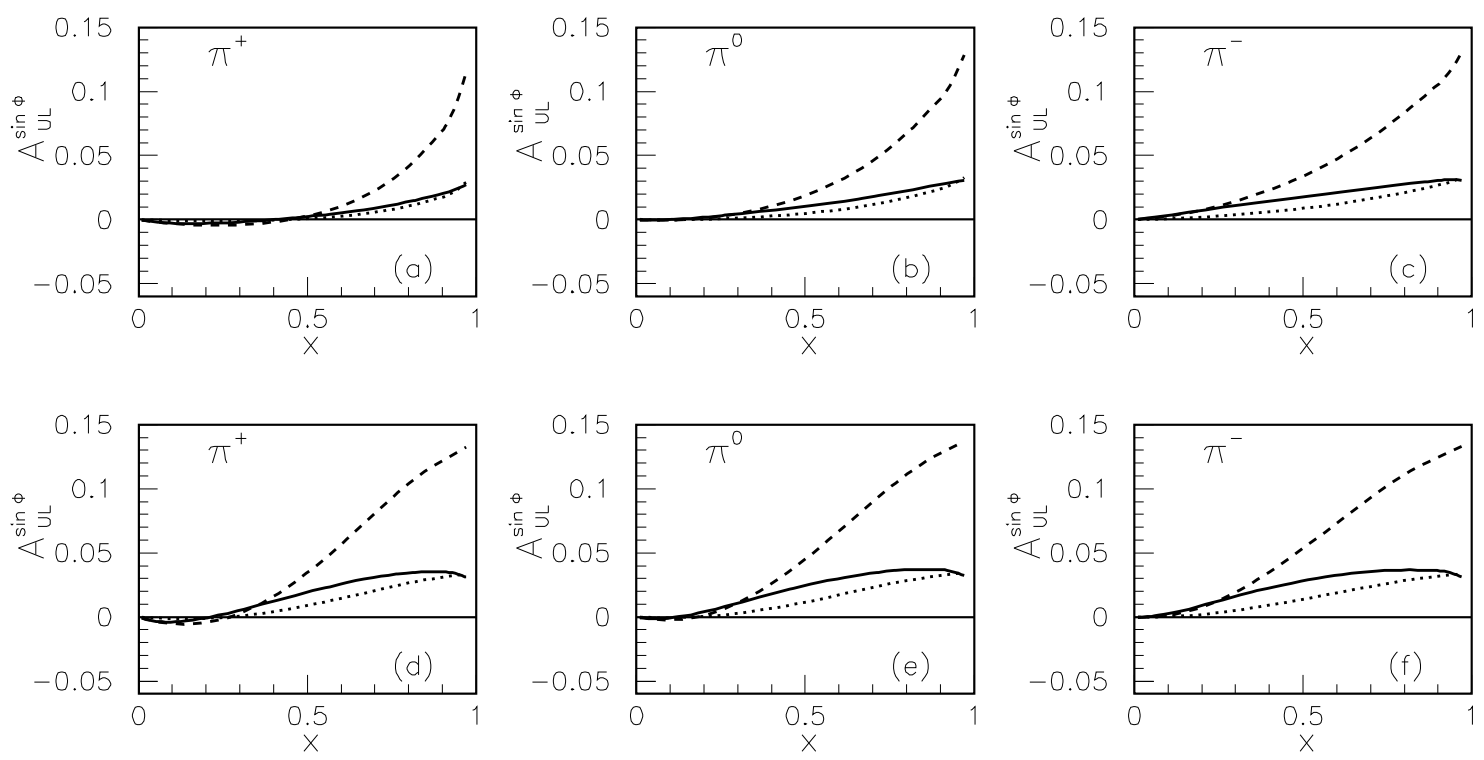

Figure 7: The same as Fig. 6, but with the neutron target.

quark diquark model and the pQCD based analysis. In order to understand this behavior, we consider the $\pi^{+}$production for a neutron target, shown in Fig. 7 (a) and (d), and compare them with the $\pi^{-}$production for a proton target, shown in Fig. 2 (c) and (f). For the proton case, the favored fragmentation process is $d \rightarrow \pi^{-}$, while the unfavored process is $u \rightarrow \pi^{-}$. The unfavored process is important due to the dominance of $u$ quark distribution over $d$ inside the proton target at large $x$. For the neutron case, the favored fragmentation process is $u \rightarrow \pi^{+}$, while the unfavored process is $d \rightarrow \pi^{+}$. The unfavored process is suppressed by a factor of 4 (from the squared charge) compared to the corresponding process of the proton target, while the favored process is enhanced by a factor of 4 compared to the corresponding process of the proton target. Thus the favored process $u \rightarrow \pi^{+}$relative to the unfavored process $d \rightarrow \pi^{+}$for the neutron target is enhanced by a factor of 16 compared to the corresponding favored process $d \rightarrow \pi^{-}$relative to the unfavored process $u \rightarrow \pi^{-}$for the proton. This implied that the favored process of $\pi^{+}$production for the neutron target plays a more important role than the corresponding process of $\pi^{-}$production for the proton target, where the unfavored process is more important and cannot be 
neglected. Thus we suggest to distinguish between the quark diquark model and the pQCD based analysis by measuring $\pi^{+}$production from a neutron target. The $\pi^{+}$ production for a neutron target is sensitive to the $u$ quark transversity distribution of the neutron, which is essentially the $d$ quark transversity distribution of the proton. Thus combination of pion production from both the proton and neutron targets can measure the flavor structure of the quark transversity distributions.

\subsection{The azimuthal asymmetries of $A_{U L}^{\sin 2 \phi}$}

For the azimuthal asymmetries with weighting function $\sin 2 \phi$, i.e., $A_{U L}^{\sin 2 \phi}$, there is only one quark distribution function $h_{1 L}^{\perp(1)}(x)$ involved in the numerator of (11). In both the Leading Approach and Approach 1, this distribution function is assumed to be zero. Therefore the azimuthal asymmetries $A_{U L}^{\sin 2 \phi}$ can only be calculated in Approach 2, and we present the results for the proton, deuteron, and neutron targets in Figs. 8, 9, and 10, respectively, in the two different models of quark transversity distributions. We find that the magnitude of these asymmetries are not so small in the two models, thus it is possible to measure them with high precision measurements. In the case of a proton target, the calculated results are similar for the two models with the available HERMES kinematical cuts, and they are both compatible with the available HERMES data. The calculated results are also similar in the two models in the case of the deteron target. However, larger differences between the predictions of the two models is found in the case of $\pi^{+}$production from a neutron target. Thus precision measurement of $A_{U L}^{\sin 2 \phi}$ from nucleon targets may provide us with information on the distribution function $h_{1 L}^{\perp(1)}(x)$ and its flavor structure. 

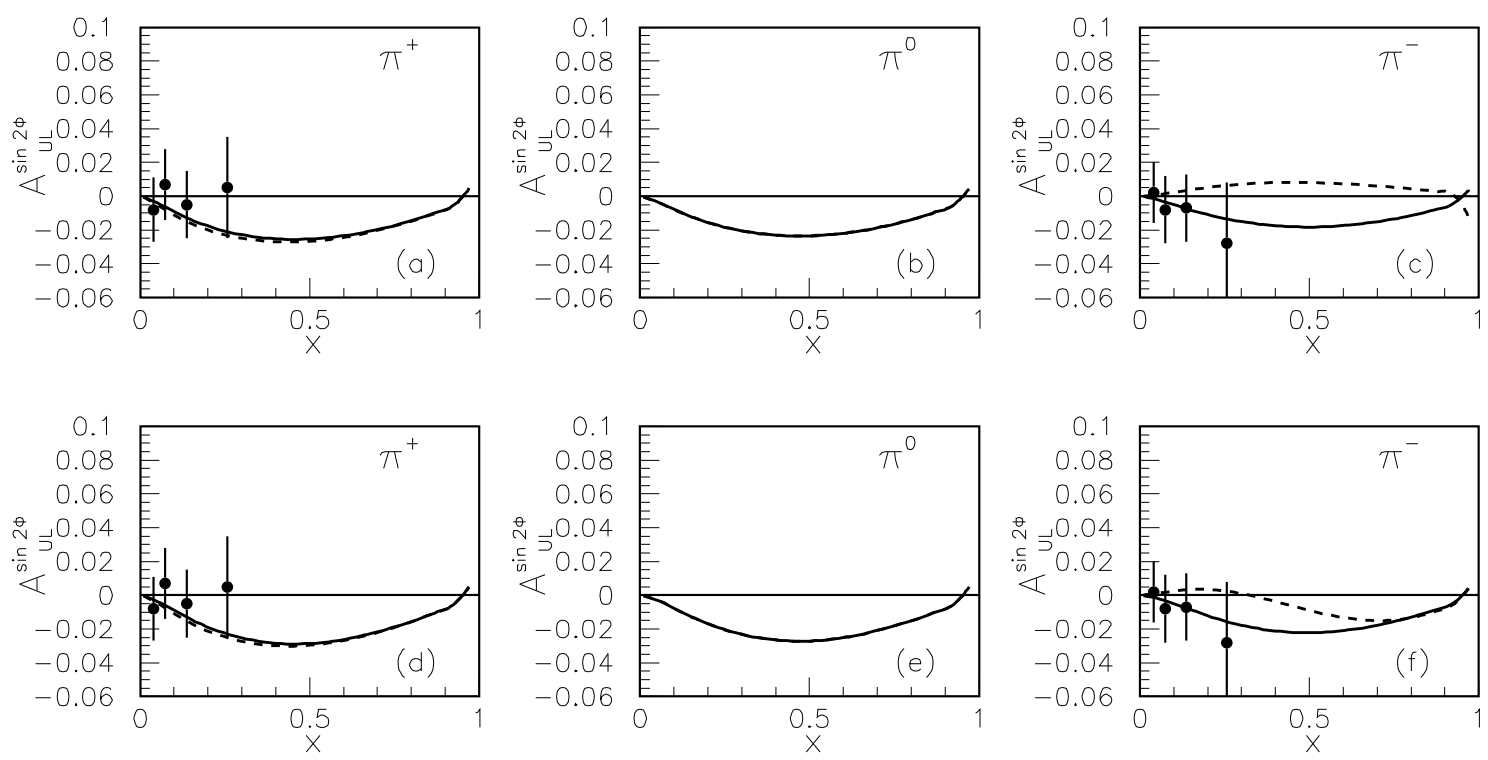

Figure 8: The azimuthal asymmetries $A_{U L}^{\sin 2 \phi}$ for semi-inclusive $\pi^{+}, \pi^{0}$, and $\pi^{-}$production in deep inelastic scattering of unpolarized positron on the longitudinally polarized proton target (with polarization $S=0.86$ ). The upper row corresponds to (a) $\pi^{+}$, (b) $\pi^{0}$, and (c) $\pi^{-}$in the quark diquark model, and the lower row corresponds to (d) $\pi^{+}$, (e) $\pi^{0}$, and (f) $\pi^{-}$in the pQCD based analysis. The solid and dashed curves correspond to the calculated results for Case 1 with both favored and unfavored fragmentation and Case 2 with only favored fragmentation, respectively.

\section{Prediction of Pion Azimuthal Asymmetries for Transversely Polarized Target}

From the above discussions, we find that although the azimuthal asymmetries off longitudinally polarized target may serve as a measurement of the transversity distributions, there are still some contribution from several unmeasured distribution functions and fragmentation functions. Their contribution are not negligible in the HERMES kinematical region, and also there are some ambiguities concerning the size of their contribution from different assumptions, as we can see from the different predictions between Approach 1 and Approach 2 in the last section. Therefore it is necessary to look for more clean processes for a direct measurement of the quark transversity. In fact, it has been known that the azimuthal asymmetries off transversely polarized target are directly connected to the Leading Approach term we discussed for the 

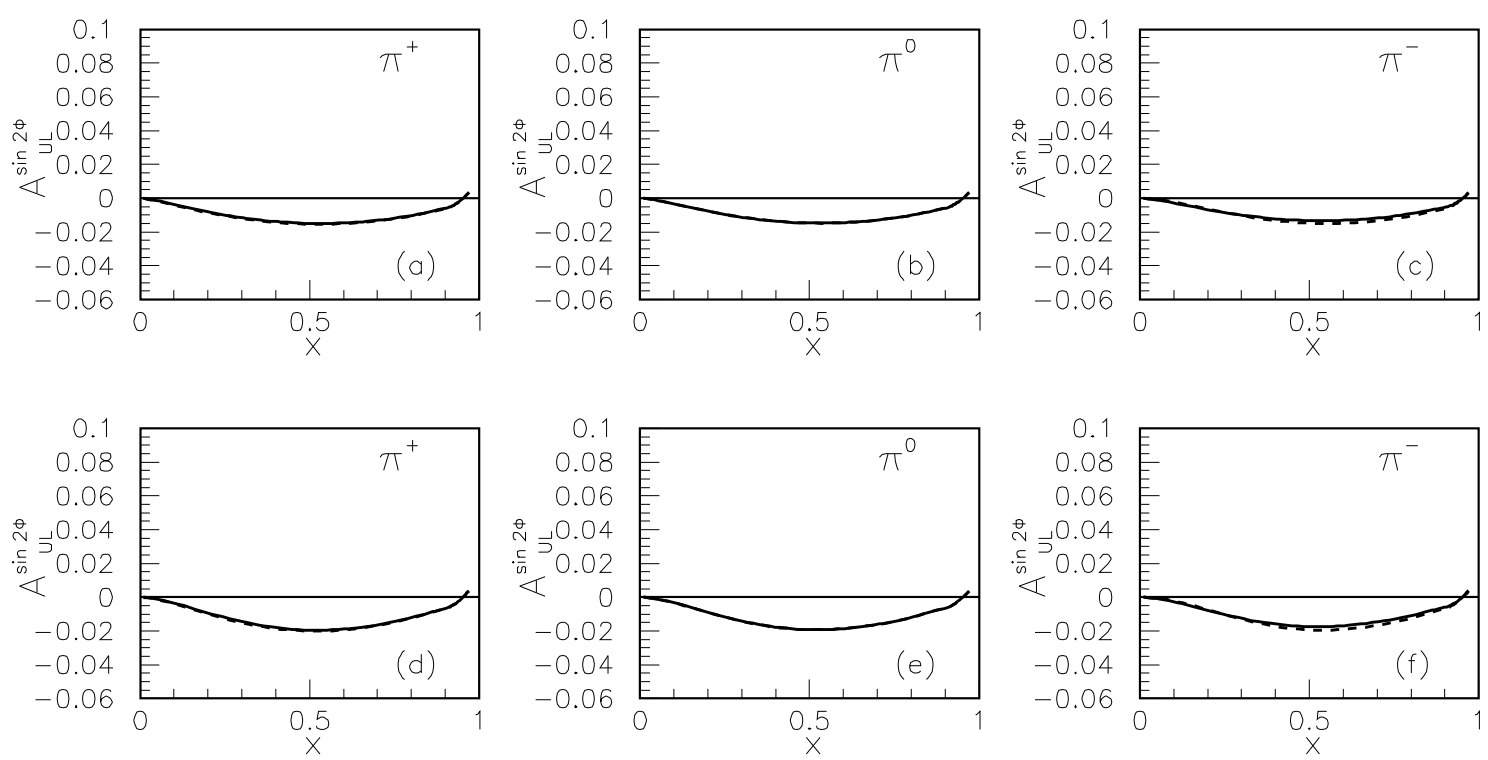

Figure 9: The same as Fig. 8, but for the deuteron target with polarization $S=0.75$.

longitudinally polarized situation. There has been some preliminary results from the SMC experiment [43], indicating evidence of non-zero azimuthal asymmetry for pion production from a transversely polarized target. Also the HERMES collaboration is planning to measure the azimuthal asymmetries of pion production from transversely polarized targets in the near future [23]. It is thus necessary to make clear predictions on the quantities they will measure by using the two sets of transversity distributions given by the quark diquark model and the pQCD based analysis.

More specifically, for an unpolarized lepton beam and a transversely polarized target, the following weighted asymmetry provides access to the quark transversity distribution [10

$$
A_{T}(x, y, z)=\frac{\int \mathrm{d} \phi^{l} \int \mathrm{d}^{2} P_{h \perp} \frac{\left|P_{h \perp}\right|}{z M_{h}} \sin \left(\phi_{s}^{l}+\phi_{h}^{l}\right)\left(\mathrm{d} \sigma^{\uparrow}-\mathrm{d} \sigma^{\downarrow}\right)}{\int \mathrm{d} \phi^{l} \int \mathrm{d}^{2} P_{h \perp}\left(\mathrm{d} \sigma^{\uparrow}+\mathrm{d} \sigma^{\downarrow}\right)},
$$

where $\uparrow(\downarrow)$ denotes target up (down) transverse polarization. The azimuthal angles are defined in the transverse space giving the orientation of the lepton plane $\left(\phi^{l}\right)$ and the orientation of the hadron plane $\left(\phi_{h}^{l}=\phi_{h}-\phi^{l}\right)$ or spin vector $\left(\phi_{s}^{l}=\phi_{s}-\phi^{l}\right)$ with respect to the lepton plane. The asymmetry (46) can be calculated from the 

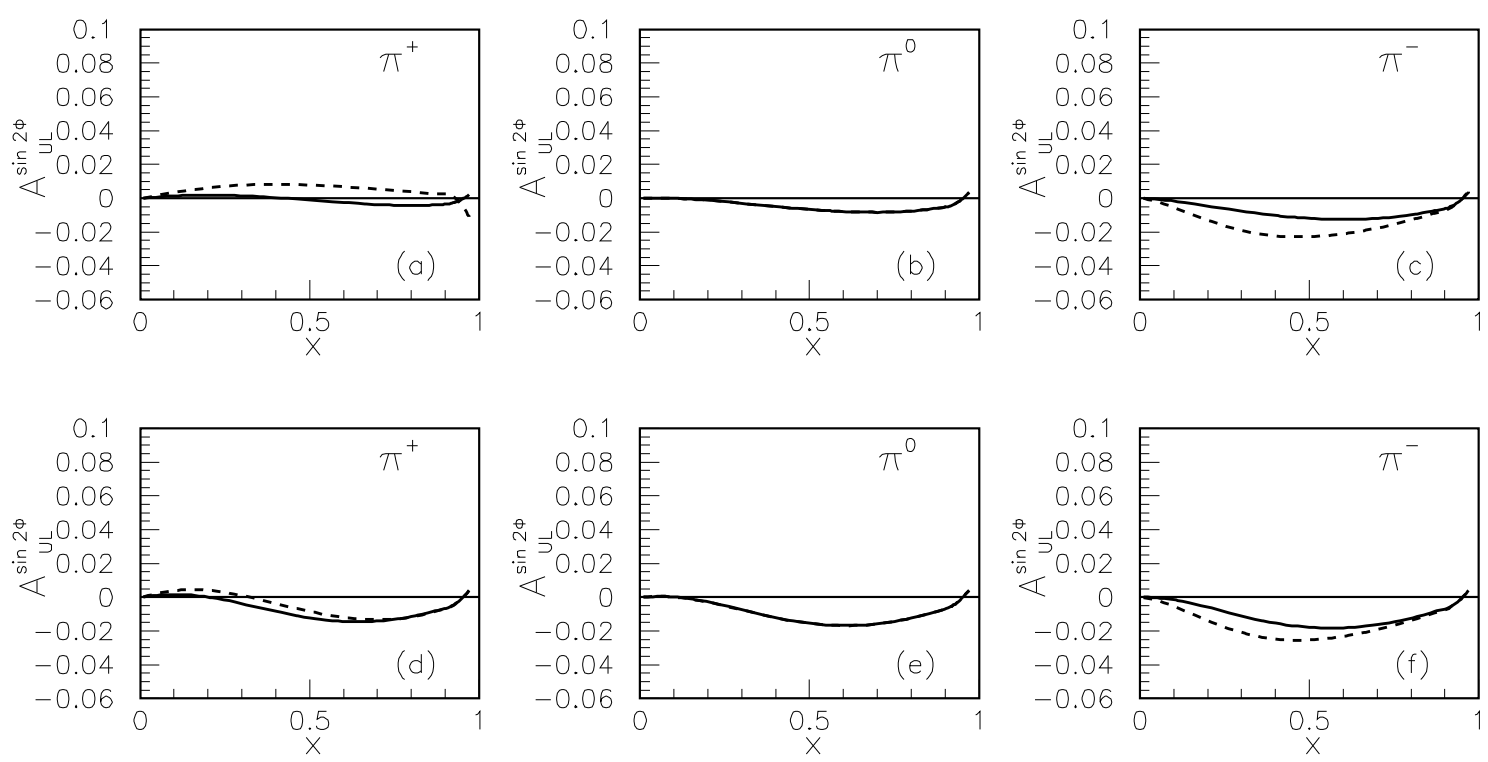

Figure 10: The same as Fig. 8, but for the neutron target with polarization $S=0.75$.

distribution functions and fragmentation functions by 10

$$
A_{T}(x, y, z)=S_{T} \frac{(1-y) \sum_{q} e_{q}^{2} \delta q(x) H_{1}^{\perp(1) q}(z)}{\left(1-y+y^{2} / 2\right) \sum_{q} e_{q}^{2} q(x) D_{1}^{q}(z)},
$$

where $S_{T}$ is the target polarization. Assuming $S_{T}=0.75$ and the same kinematical cuts as in the MERMES experiments, and with all of the quantities discussed as before, we can calculate the asymmetry $A_{T}$ with the two different sets of quark transversity distributions. The integration over $y$ and $z$ should be performed for both the numerator and denominator. The predictions for a proton target are given in Fig.11, from where we notice that the azimuthal asymmetries of $\pi^{+}, \pi^{0}$, and $\pi^{-}$ production from a proton target are mainly controlled by the $u$ quark transversity distribution, and they can serve to measure $\delta u(x)$. The predictions for pion production from a deuteron target, given in Fig. 12, are also found to have small differences between the two different models. However, the azimuthal asymmetries from a neutron target are predicted to have a large difference between the quark diquark model and the pQCD based analysis, as shown in Fig. 13. Thus they can serve to measure the flavor structure of quark transversity distributions, i.e., one can measure both $\delta u(x)$ and $\delta d(x)$ by using the azimuthal asymmetries from both proton and neutron 
targets.
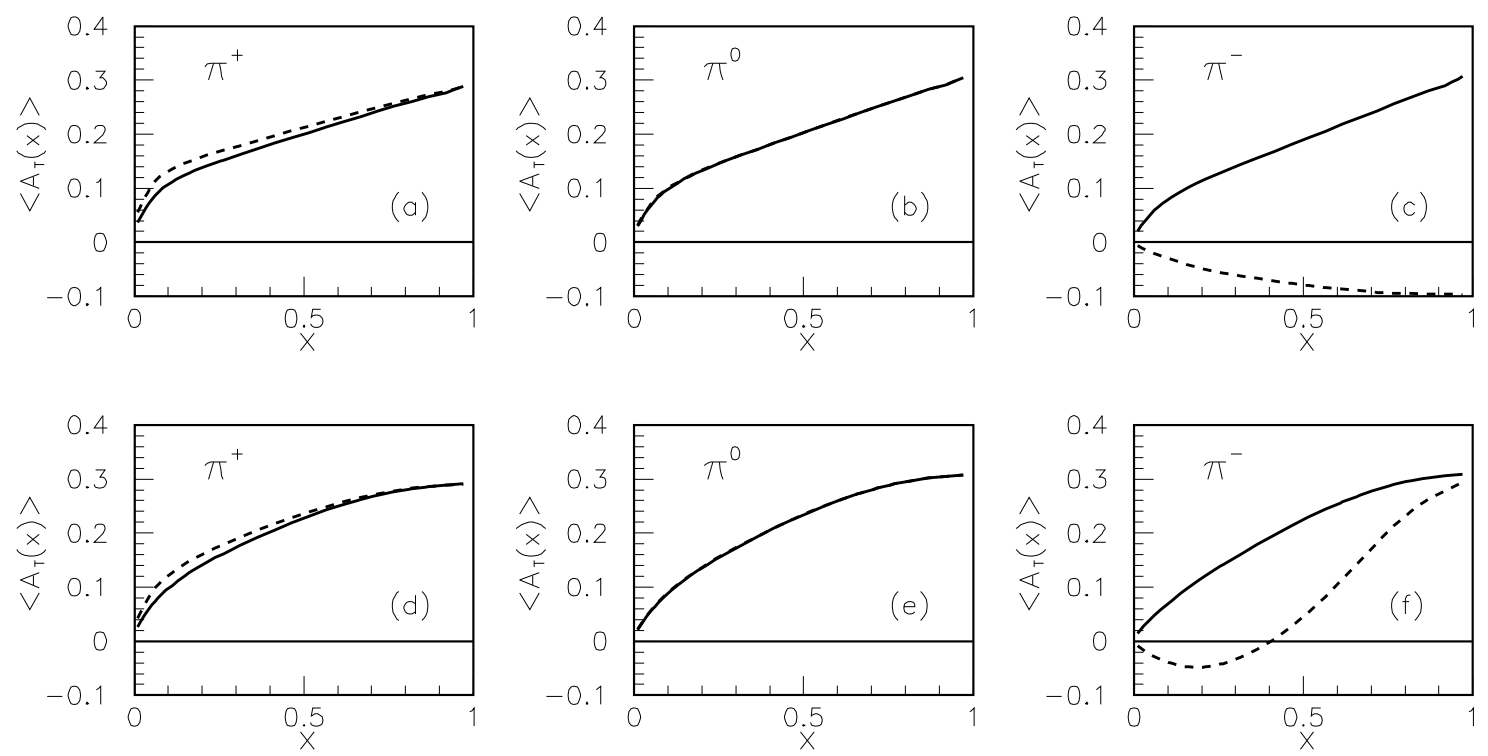

Figure 11: The azimuthal asymmetries $\left\langle A_{T}(x)\right\rangle$ for semi-inclusive $\pi^{+}, \pi^{0}$, and $\pi^{-}$ production in deep inelastic scattering of unpolarized positron on the transversely polarized proton target. The upper row corresponds to (a) $\pi^{+}$, (b) $\pi^{0}$, and (c) $\pi^{-}$ in the quark diquark model, and the lower row corresponds to (d) $\pi^{+}$, (e) $\pi^{0}$, and (f) $\pi^{-}$in the pQCD based analysis. The solid and dashed curves correspond to the calculated results for Case 1 with both favored and unfavored fragmentation and Case 2 with only favored fragmentation, respectively.

\section{Summary and Conclusions}

In this paper, we analyzed in detail the azimuthal spin asymmetries of pion production, in semi-inclusive deep-inelastic scattering of unpolarized charged lepton beams on longitudinally and transversely polarized nucleon targets. Various assumptions and approximations of the distribution functions and fragmentation functions were examined. It was found that different approaches of distribution functions and fragmentation functions lead to different predictions of the azimuthal asymmetries in the available HERMES kinematical region. This means that the effects from unknown 

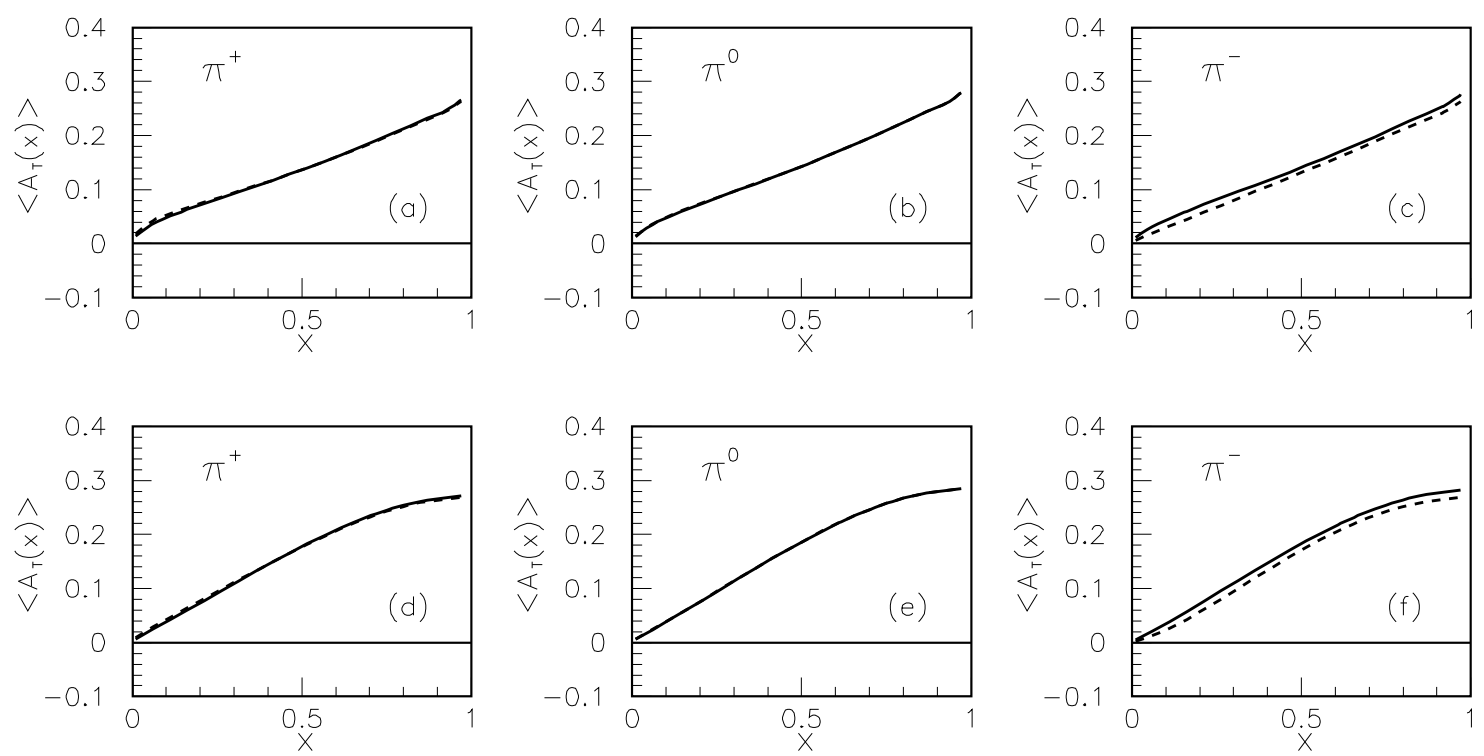

Figure 12: The same as Fig. 11, but for the deuteron target.

distribution functions may have sizable effects on the azimuthal asymmetries of the HERMES experiments, thus one needs to consider them before attributing the HERMES data as a measurement of transversity distributions. We also found that the predictions of $\pi^{-}$production from the proton target are quite different for two cases of both favored and unfavored fragmentation, and of only favored fragmentation. This means that the unfavored fragmentation functions play an important role for $\pi^{-}$production from a proton target, due to the dominance of $u$ quarks inside the proton target, thus the unfavored $u \rightarrow \pi^{-}$fragmentation are not negligible. This point was not considered in the available studies of azimuthal asymmetries. The pion production from the proton target is most suitable to study the $u$ quark transversity distribution. In combination with the pion production from a neutron target, it is possible to measure both of the $u$ and $d$ quark transversity distributions. Thus we suggest to measure the azimuthal symmetries of pion production from the neutron target, in order to test different model predictions. Predictions of azimuthal asymmetries of pion production in semi-inclusive deep-inelastic scattering of unpolarized charged lepton beams on transversely polarized nucleon targets are also given, 

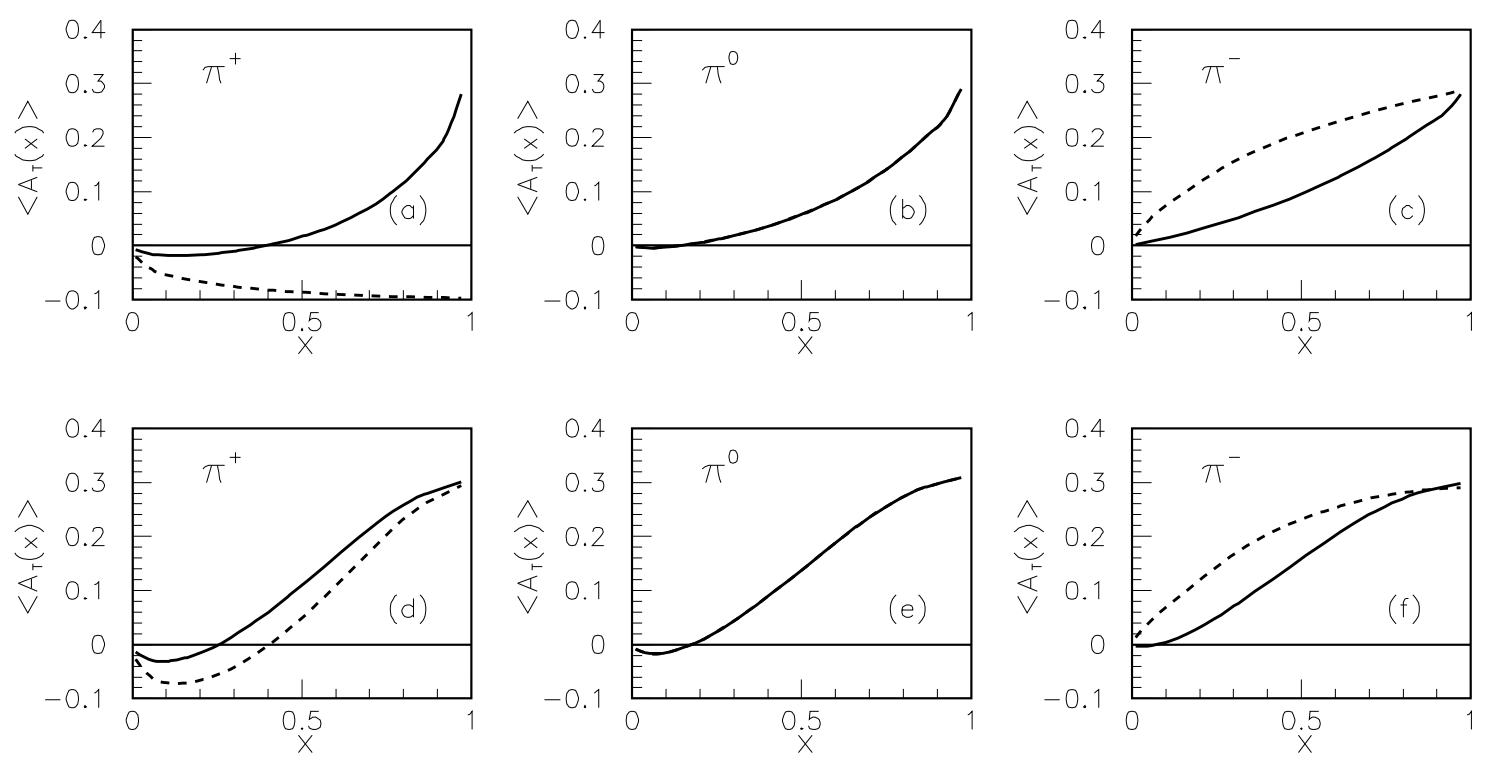

Figure 13: The same as Fig. 11, but for the neutron target.

and different predictions between two different models are given for pion production from a neutron target. This study will be useful for designing experiments aiming at measuring transversity distributions through pion production in semi-inclusive deepinelastic scattering of unpolarized beams on longitudinally and transversely polarized targets. It is also useful for theoretical studies aiming at extracting transversity distributions from experimental observation of azimuthal asymmetries of pion production in semi-inclusive deep-inelastic scattering.

Acknowledgments: This work is partially supported by National Natural Science Foundation of China under Grant Numbers 19975052, 19875024, and 10025523, by Fondecyt (Chile) postdoctoral fellowship 3990048, and by Fondecyt (Chile) grant 8000017. 


\section{References}

[1] HERMES Collaboration, A. Airapetian et al., Phys. Rev. Lett. 84, 4047 (2000).

[2] HERMES Collaboration, A. Airapetian et al., hep-ex/0104005, to appear in Phys. Rev. D , (2001).

[3] For a recent review, see, e.g., V. Barone, A. Drago, and P.G. Ratcliffe, hepph/0104283, to appear in Phys. Rep. , (2001).

[4] For a review, see, e.g., J.F. Owens and W.-K. Tung, Ann. Rev. Nucl. Part. Sci. 42, 291 (1992).

[5] For a review, see, e.g., P.L. McGaughey, J.M. Moss, and J.C. Peng, Ann. Rev. Nucl. Part. Sci. 49, 217 (1999).

[6] J. Collins, Nucl. Phys. B 396, 161 (1993).

[7] A.M. Kotzinian, Nucl. Phys. B 441, 234 (1995).

[8] M. Anselmino, B. Boglione, and F. Murgia, Phys. Lett. B 362, 164 (1995).

[9] P.J. Mulders and R.D. Tangerman, Nucl. Phys. B 461, 197 (1996).

[10] A.M Kotzinian and P.J. Mulders, Phys. Lett. B 406, 373 (1997).

[11] R. Jaffe, X. Jin, and J. Tang, Phys. Rev. Lett. 80, 1166 (1998).

[12] R.D. Tangerman and P.J. Mulders, Phys. Rev. D 51, 3357 (1995); D. Boer and R. Tangerman, Phys. Lett. B 381, 305 (1996); D. Boer and P.J. Mulders, Phys. Rev. D 57, 5780 (1998); D. Boer, R. Jakob, and P.J. Mulders, Phys. Lett. B 424, 143 (1998).

[13] A.M. Kotzinian et al., Nucl. Phys. A 466\&467, 290c (2000).

[14] A.V. Efremov, K. Goeke, M.V. Polyakov, and D. Urbano, Phys. Lett. B 478, 94 (2000). 
[15] A. Schäfer and O.V. Teryaev, Phys. Rev. D 61, 077903 (2000).

[16] D. Boer, RIKEN Rev. 28, 26 (2000), hep-ph/9912311.

[17] M. Boglione and P.J. Mulders, Phys. Lett. B 478, 114 (2000).

[18] M. Anselmino and F. Murgia, Phys. Lett. B 483, 74 (2000).

[19] K. Suzuki, RIKEN Rev. 28, 105 (2000), hep-ph/0002218.

[20] B.-Q. Ma, I. Schmidt, and J.-J. Yang, Phys. Rev. D 63, 037501 (2001).

[21] K.A. Oganessyan, N. Bianchi, H.R. Avakian, A.M. Kotzinian, hep-ph/9808368.

[22] E. De Sanctis, W.-D. Nowak, and K.A. Oganessyan, Phys. Lett. B 483, 69 (2000).

K.A. Oganessyan, N. Bianchi, E. De Sanctis, W.-D. Nowak, hep-ph/0010261.

[23] V.A. Korotkov, W.-D. Nowak, and K.A. Oganessyan, hep-ph/0002268, Euro. Phys. J. C 18, 639 (2001).

[24] A.V. Efremov, K. Goeke, P. Schweitzer, hep-ph/0108213.

[25] R. Jaffe and X. Ji, Nucl. Phys. B 375, 527 (1992).

[26] R.D. Tangerman and P.J. Mulders, hep-ph/9408305.

[27] R.P. Feynman, Photon Hadron Interactions (Benjamin, New York, 1972), p. 150.

[28] F.E. Close, Phys. Lett. 43 B, 422 (1973); Nucl. Phys. B 80, 269 (1974); R. Carlitz, Phys. Lett. B 58, 345 (1975); J. Kaur, Nucl. Phys. B 128, 219 (1977); A. Schäfer, Phys. Lett. B 208, 175 (1988); F.E. Close and A.W. Thomas, ibid B 212, 227 (1988); N. Isgur, Phys. Rev. D 59, 034013 (1999).

[29] B.-Q. Ma, Phys. Lett. B 375, 320 (1996).

[30] G.R. Farrar and D.R. Jackson, Phys. Rev. Lett. 35, 1416 (1975). 
[31] R. Blankenbecler and S.J. Brodsky, Phys. Rev. D 10, 2973 (1974); J.F. Gunion, ibid. D 10, 242 (1974); S.J. Brodsky and G.P. Lepage, in Proc. 1979 Summer Inst. on Particle Physics, SLAC (1979).

[32] S.J. Brodsky, M. Burkardt, and I. Schmidt, Nucl. Phys. B 441, 197 (1995).

[33] B.-Q. Ma, I. Schmidt, J. Soffer, and J.-J. Yang, Phys. Rev. D 64, 014017 (2001).

[34] B.-Q. Ma, I. Schmidt, and J. Soffer, Phys. Lett. B 441, 461 (1998).

[35] B.-Q. Ma and I. Schmidt, J. Phys. G 24, L71 (1998); Phys. Rev. D 58, 096008 (1998).

[36] B.-Q. Ma, J. Phys. G 17, L53 (1991); B.-Q. Ma and Q.-R. Zhang, Z. Phys. C 58, 479 (1993).

[37] I. Schmidt and J. Soffer, Phys. Lett. B 407, 331 (1997).

[38] S. Kretzer, E. Leader, and E. Christova, hep-ph/0108055.

[39] V.N. Gribov and L.N. Lipatov, Phys. Lett. B 37, 78 (1971); Sov. J. Nucl. Phys. 15, 675 (1972); S.J. Brodsky and B.-Q. Ma, Phys. Lett. B 392, 452 (1997).

[40] J. Blümlein, V. Radindran and W.L. van Neerven, Nucl. Phys. B 589, 349 (2000).

[41] V. Barone, A. Drago, and B.-Q. Ma, Phys. Rev. C 62, 062201(R) (2000).

[42] CTEQ Collaboration, H. L. Lai et al., Eur. Phys. J. C 12, 375 (2000).

[43] A. Bravar, Nucl. Phys. (Proc.Suppl.) B 79, 520 (1999). 